\title{
Comparative Study between Conchal Cartilage Grafts and Split Rib Grafts in the Reconstruction of Orbital Floor Fractures
}

\author{
EMAN NAGY MOHMED, M.D.; HANY SAAD SETTA, M.D. and AHMED EL-SHAHAT, M.D. \\ The Department of Plastic Surgery, Faculty of Medicine, Ain Shams University, Egypt
}

\begin{abstract}
Background: Orbital floor fractures always represent a challenge for reconstruction. Various techniques for treatment had evolved over the past years, each with their strengths and weaknesses. The main aim of surgical repair is to relocate herniated orbital tissue and fat back into the orbit restoring both the function and aesthetic aspect. Reconstruction of orbital floor can be performed using various types of materials, either autologous as rib grafts or cartilage grafts or alloplastic as titanium mesh.

Aim of the Study: The aim of this study is to compare the results of using autologous cartilage grafts with those of split rib graft in small posttraumatic orbital floor defects reconstruction.

Patients and Methods: 30 patients with orbital floor defects due to maxillofacial trauma. They were divided into 2 groups according to the technique of reconstruction: Group 1: 15 patients who had reconstruction by onlay split rib grafts Group 2: 15 patients who had reconstruction by conchal cartilage grafts, using either unilateral or sutured bilateral conchal cartilage grafts in large defects. Both methods were analyzed clinically and radiologically and were compared as regards, their advantages and disadvantages Postoperative assessment using CT scan were used.
\end{abstract}

Results: With the conchal cartilage grafts, the results were more favorable with statistically significant decrease in complications.

Conclusion: Reconstruction of orbital floor fractures using conchal cartilage grafts represents a safe and reliable method of reconstruction with less complications.

Key Words: Orbital - Floor - Defects - Conchal-Cartilage - Splitted - Rib.

\section{INTRODUCTION}

Orbital floor fractures are of the commonest maxillofacial injuries nowadays due to increased incidence of interpersonal violence and road traffic accidents. As denoted by Chang and Manolidis, Mackenzie was the first to describe orbital floor fractures in 1844 [1]. Since then, various studies have discussed the indications and most advantageous time in which surgery should be considered as well as the most considerable surgical modality to be used for orbital blowout fractures.

Over a hundred years later, specifically in the year 1957, Smith and Regan identified inferior rectus muscle entrapment which resulted in diminished ocular motility during orbital fractures and named them "blow-out fractures" [2]. Since then, multiple studies have researched the indications and most appropriate timing for surgical intervention in addition to the most favorable surgical modality [3].

In order to use autografts, autologous patient tissue must be available for harvest from a donor site. It should be further shaped to perfectly match the dimensions of the defect in order to provide with structurally intact support for the underlying tissues and structures. It is usually easier to harvest cartilage and shape it, hence further contributes to long-term support without resorption [4,5].

Autologous reconstruction of orbital floor by cartilage grafts was used in different studies as presented by Castellani et al., [6] and Ozayzgan et al., [7].

However other previous studies have considered bone grafts for the orbital floor as the recommended management for orbital fracture. This approach is based on the availability of adequate amount of autologous bone obtained from a donor site. It has to be further shaped and inserted in order to give firm structural support in reestablishing the defect. Being biocompatible, craniofacial surgeons have favored bone grafts in management of such fractures. Among the donor sites are the split calvarial Bone Graft, rib, and iliac crest [8-10]. Grafts could be either located as onlay Grafts [11], fixated with a plate and screw [12], fixated with a lag screw or fixated in conjunction with an alloplastic material, such as titanium mesh or porous polyethylene 
sheets [13-15]. There is no definite approval about the suitable material for reconstruction.

The aim of this study is to compare the results of using autologous cartilage graft with those of split rib graft in small posttraumatic orbital floor defects reconstruction.

\section{PATIENTS AND METHODS}

A randomized controlled comparative study was conducted in the Plastic and Maxillofacial Surgery Department at Ain shams University Hospitals. The present study included 30 patients, 22 males and 8 females, with age ranging from $23-$ 49 years, average 36 years. They presented to the outpatient and emergency room of the hospital with orbital floor fractures along with floor defects either isolated or with other maxillofacial fractures from November 2014 to December 2016.

Patients were subdivided into two groups, 15 cases were managed using autologous cartilage grafts for reconstruction of the orbital floor defects either unilateral or sutured biconchal cartilage grafts according to the size of the defect (Group 1) and 15 cases were managed using onlay split rib graft (Group 2). The patients were selected randomly irrespective of age and sex.

The following inclusion criteria were fulfilled: (1) Clinical diagnosis of orbital floor defects; (2) Computed Tomography (CT) showing orbital floor defects; (3) Intraoperative finding of small orbital floor defects $\leq 2 \mathrm{~cm}^{2}$. Exclusion criteria include (1) orbital floor fracture without bone defect or (2) large defects $>2 \mathrm{~cm}^{2}$ or patients who had prior surgery were excluded from the study.

History was recorded \& full clinical examination was performed including local \& general examinations. Local examination was done paying special attention to the following: Restrained ocular movement, ocular dystopia, enophthalos, diplopia specifically on upward gaze, paresthesia in the infraorbital nerve distribution and step deformity. All patients were ophthalmologically examined on admission, preoperatively, postoperatively after the swelling had ceased, and during follow-up. Any other injuries were recorded and assessed and confirmed by related different specialties.

All patients had CT imaging with axial, coronal and sagittal cuts and 3D image construction. Informed consent was obtained from all patients. All the patients had their operations under general anesthesia. We used transconjunctival incision/ subciliary incisions in all cases. Greatest dimensions of the defect are measured and according to the size of the defect In Group 1, defects of orbital floor were reconstructed by Conchal Cartilage Grafts either unilateral (defect size up to $1.5 \mathrm{~cm}^{2}$ ) or sutured biconchal cartilage grafts (defect size $\left.1.5-2 \mathrm{~cm}^{2}\right)$.

Conchal cartilage grafts were harvested through anterior or posterior incision. Special care was in concern during harvesting the graft to ensure the integrity of the concha as monoblock aiming for better occlusion of the floor.

In Group 2, similar defects were reconstructed using onlay Rib Grafts, where the rib was harvested, splitted and applied to the defect.

In all cases all the prolapsed orbital contents (preorbital fat, inferior rectus muscle and inferior oblique muscle) were freed from the maxillary sinus and free eye movement was ensured. After grafting, closure was done according to planes of access to the orbit with careful closure of periosteum to avoid extrusion of the applied graft.

Postoperative patient care was done including medical treatment in the form of intravenous fluids, analgesics, antibiotics and postoperative followup CT. Moreover, ophthalmology examination was a routine. Examination to our patients was done at our outpatient clinic at 1, 3 and 6 months postoperatively. Digital photos were taken at the time of injury, pre-and post-operative and with followup visits. Complications if any were noted for further assessment and management.

\section{RESULTS}

The most common preoperative complaints were diplopia in 18 patients $(60 \%)$ and parathesia of the ipsilateral infraorbital nerve in 12 patients (40\%). Concerning the etiology of fractures were recorded MCA was the causative factor in 18 cases (60\%), fights and direct trauma in 12 cases (40\%).

Four cases had temporarily scleral show postoperatively ( 2 cases in Group 1 and 2 cases in Group 2) and were managed conservatively. Seven cases had diplopia (two in Group 1 and five in Group 2), which improved completely within 6 months postoperatively. By palpation the onlay Rib Graft was felt (Group 2) in three patients at the edge of the infraorbital rim. No complications to the donor site were observed. Commonly occurring complications such as hemorrhage, infection and wound disruption were not present in the outcome of this study. All patients were satisfied with their postoperative outcome with significant 
improvement in esthetic appearance, and return to normal life activities within three weeks. Symmetry was restored among the injured and uninjured sides.

All results were statistically analyzed using IBM SPSS22 program (2-paired $t$-test) where all collected data showed no statistical significance concerning age, sex, mode of trauma and the size of the defect relative to the method of reconstruction. Whereas concerning complications, there was a higher statistical significance for reconstruction by conchal cartilage graft compared to reconstruction by split rib graft.

Table (1) shows that there is no statistical significant difference as regards age, sex, mode of trauma and size of the defect. Whereas there is a highly significant relation between the rate of complications and the technique of reconstruction, the $p$-value of the conchal cartilage graft "group 1 " $(p=0.00)$ is of higher significance than the $p$ value $(p=0.02)$ of the onlay split rib graft "group $2 "$.

Table (1): Comparing the two methods of Orbital floor reconstruction by conchal cartilage graft and onlay split rib graft.

\begin{tabular}{lccccccc}
\hline & \multicolumn{3}{c}{$\begin{array}{c}\text { Group (1) Conchal } \\
\text { CartilageGraft }\end{array}$} & & \multicolumn{3}{c}{$\begin{array}{c}\text { Group (2) } \\
\text { Onlay Rib Graft }\end{array}$} \\
\cline { 2 - 3 } & $\begin{array}{c}\text { Mean } \\
(\mathrm{cm})\end{array}$ & $\begin{array}{c}\text { Std. } \\
\text { Deviation }\end{array}$ & $\begin{array}{c}\text { Sig. } \\
\text {-value }\end{array}$ & & $\begin{array}{c}\text { Mean } \\
(\mathrm{cm})\end{array}$ & $\begin{array}{c}\text { Std. } \\
\text { Deviation }\end{array}$ & $\begin{array}{c}\text { Sig. } \\
p \text {-value }\end{array}$ \\
\hline Age & 38.60 & 6.30 & 0.1 & & 35.13 & \pm 5.68 & 0.12 \\
Sex & 0.33 & \pm 0.48 & 0.1 & & 0.37 & \pm 0.45 & 0.2 \\
Mode of trauma & 0.53 & \pm 0.64 & 0.2 & & 0.46 & \pm 0.74 & 0.3 \\
Size of Defect & 0.67 & \pm 0.22 & 0.1 & & 0.17 & \pm 0.27 & 0.3 \\
Complications & 3.46 & \pm 1.41 & 0.00 & 2.06 & \pm 1.53 & 0.02 \\
\hline
\end{tabular}

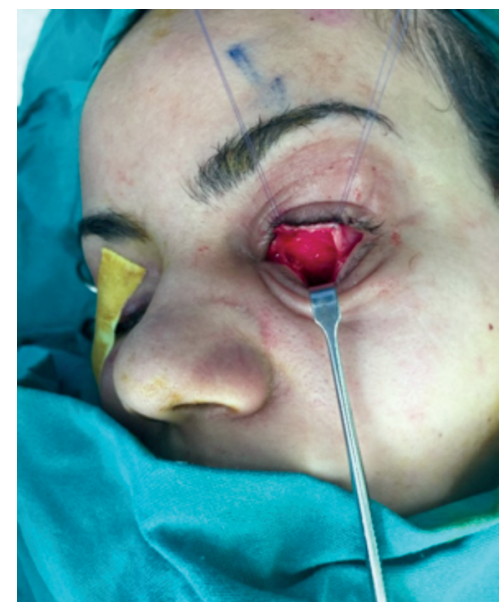

Fig. (1A): The defect after transconjunctival incision.

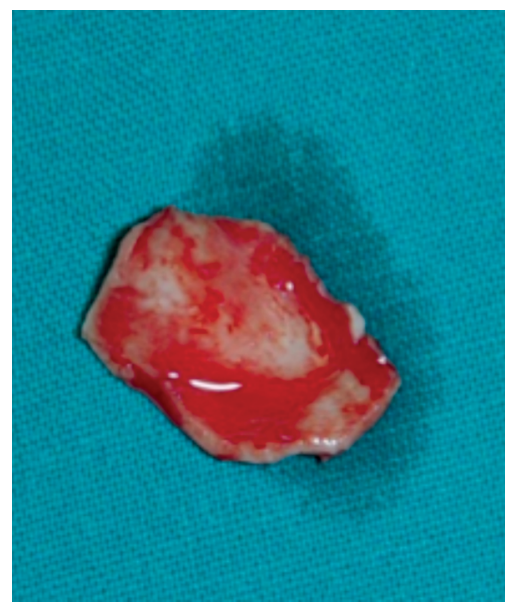

Fig. (1C): Conchal Cartilage Graft.

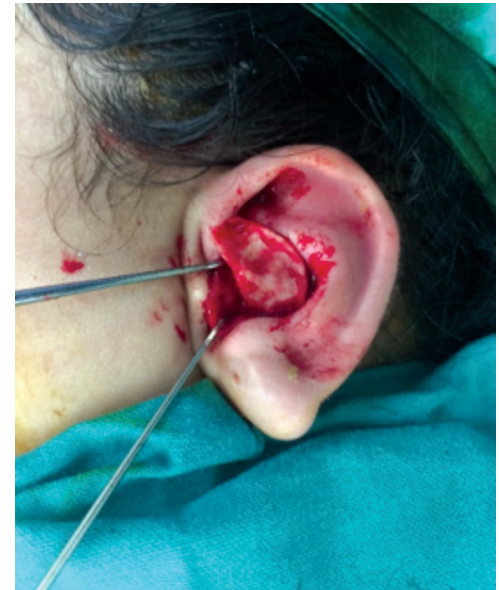

Fig. (1B): Harvesting Conchal Cartilage Graft.

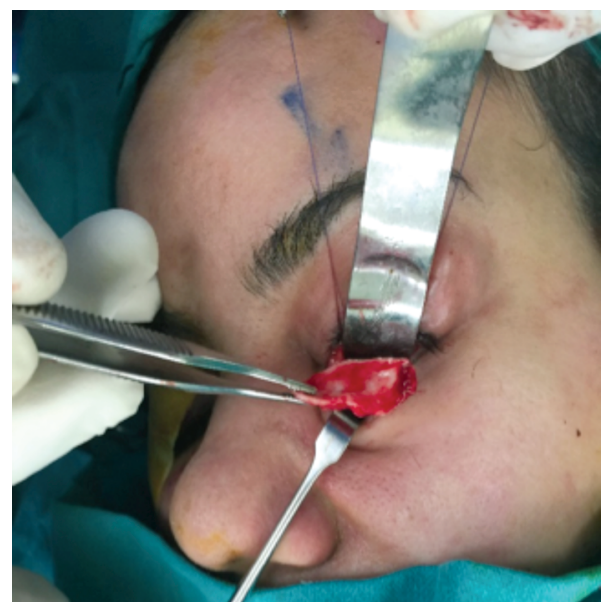

Fig. (1D): Conchal Cartilage Graft application. 


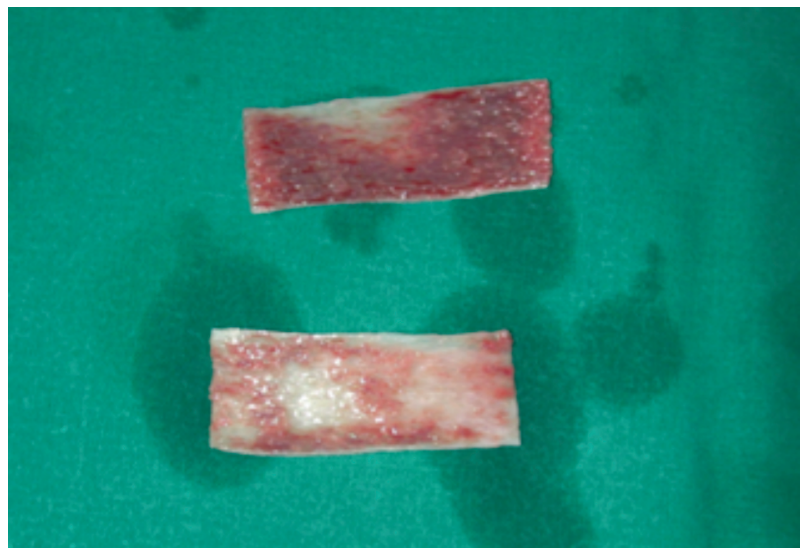

Fig. (2A): Onlay Rib Graft.

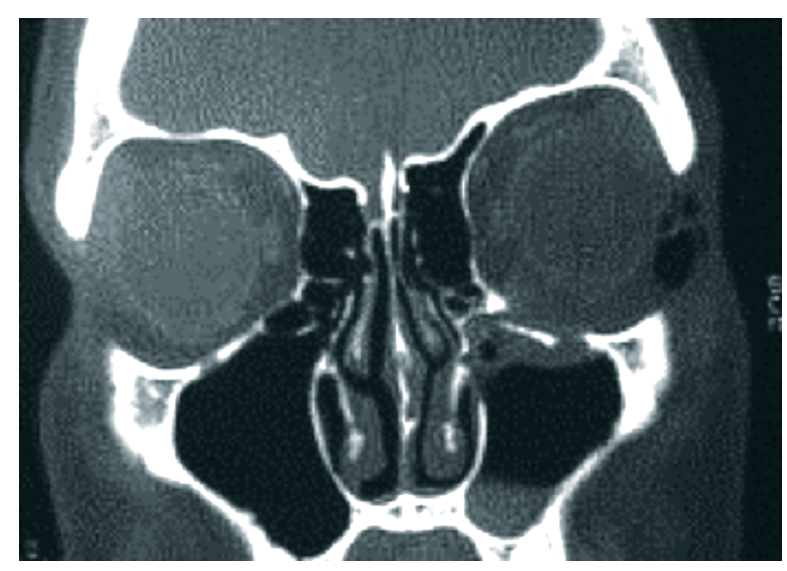

Fig. (3A): Preoperative (A) of Coronal CT defect of left orbital floor fracture.

\section{DISCUSSION}

Treatment of the orbital floor fractures is a controversial issue concerning the reconstructive material. Different materials are used either autologous or alloplastic materials. In our study, the choice of autologous materials was motivated by the low cost, low morbidity and their biocompatibility. In this study, we compared the results of reconstruction of posttraumatic orbital floor defects by autologous cartilage grafts and onlay rib grafts.

Due to the easy access for harvest and the possibility to shape, cartilage has the ability to support the surrounding tissues without resorption [4]. There are many sources for autogenous cartilage, one of which is the auricular concha. It is readily available for repair of orbital floor defects [16]. Constaintian in 1982 used conchal cartilage for repair of orbital defects $<2 \mathrm{~cm}^{2}$ and vouched for graft tailoring with ease due to the natural curve that nicely fits the orbital floor [17].

According to Bayat et al., being an abundant and accessible autogenous source that can easily

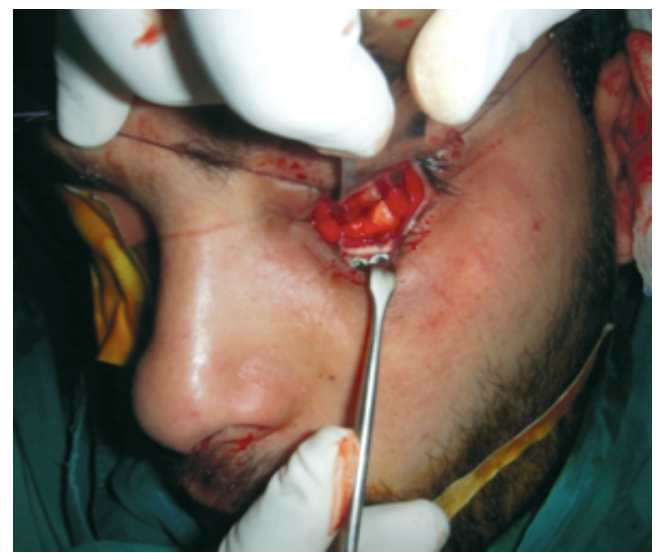

Fig. (2B): Using Onlay Rib Graft in the reconstruction of orbital floor defect.

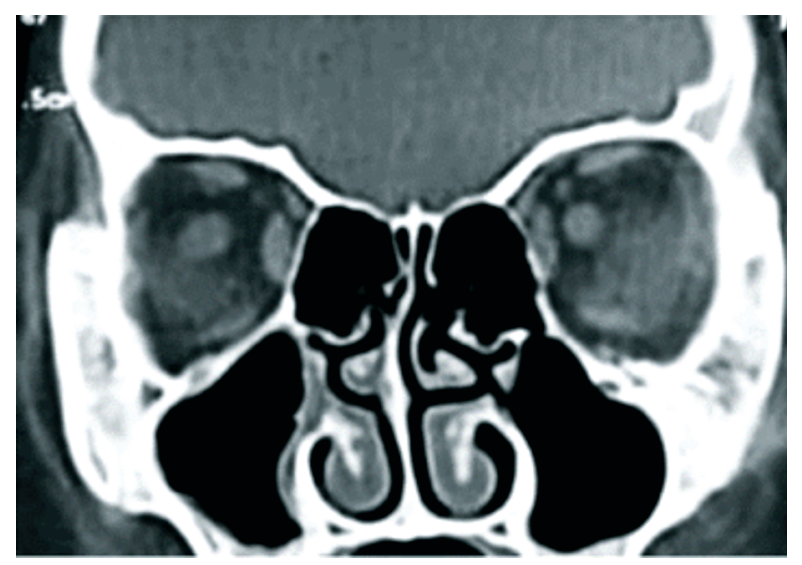

Fig. (3B): Postoperative (B) of Coronal CT defect of left orbital floor fracture reconstructed by cartilage graft.

support the orbital floor with minimal morbidity renders cartilage perfect as a grafting material. All this and more makes it questionable to why it is not regularly utilized as a reconstruction material [18].

Also Talesh et al., [19] and Ozyazgan et al., [7] in their studies recommended the use of cartilage grafts for reconstruction of orbital floor defects, due to its ideal flexibility, satisfactory resistance supporting the orbital contents.

In our study, Group 1 had comparable results to other studies with statistical significance concerning the rateof complications $(p=0.00)$ Compared to the other group in our study. Moreover low morbidity of donor site and no aesthetic deformity postoperatively matches well with the results of other studies $[\mathbf{1 0 , 1 9 ]}$.

Onlay Rib Graft, being an autologous bone, it is characterized by its strength and vascularization potential. Being an autologous source of living tissue, common side effects such as foreign body reactions including infection, formation of capsule, 
extrusion and ocular tethering are reduced [9]. Despite all the strengths of using the autologous bone graft, some unfavorable aspects need to be taken into consideration.

Mintz et al., in their study stated that bone is not that easy to yield into the needed shape and if done without care, it could easily break [20]. Lin et al., in his study showed that bone graft resorption could not be predicted or anticipated [12,21,22], the degree of which could not be priory identified.

In cases of using an autologous bone graft from a remote donor site, increase in time under general anesthesia and operating time could occur, in addition to risks related to the donor site [9]. In our study, group 2 showed comparable results with other studies using split rib grafts concerning the rate of complications and aesthetic outcome.

In this study, the results were assessed statistically to reach the conclusion that both are good reconstructive materials; but the conchal cartilage grafts has less drawbacks regarding donor site morbidity, moreover the usage of bilateral sutured conchal cartilages helped in some cases to overcome the problem of defect size reaching $2 \mathrm{Cm}^{2}$.

\section{Conclusion:}

Conchal cartilage graft is one of the most suitable autogenous graft sources for repair of defective small orbital floor fractures up to $2 \mathrm{~cm}^{2}$. It has the advantages of readily obtainable, could be adapted to the orbital floor with minimal morbidity to the donor site and adequate for reconstruction with good aesthetic outcome.

\section{REFERENCES}

1- Chang E. and Manolidis S.: Orbital floor fractures management; Facial. Plast. Surg., 21: 207-13, 2005.

2- Smith B. and Regan W.: Blow-out fracture of the orbit: Mechanism and correction of internal orbital fracture; Am. J. Ophthalmol., 6: 733-9, 1957.

3- JIn H., Yeon J., Shin S., Choi Y. and Lee D.: Endoscopic vs external repair of orbital blowout fractures; Otolaryngol. Head Neck Surg., 136: 38-44, 2007.

4- Baino F.: Biomaterials and implants for orbital floor repair, Acta Biomaterialia, 7: 3248-66, 2011.

5- D. Mok, L. Lessard, C. Cordoba, P.G. Harris and A. Nikolis.: A review of materials currently used in orbital floor reconstruction. Can. J. Plast. Surg., 12 (3): 134-140, 2004.

6- Castellani A., Negrini S., Zanetti U.: Treatment of orbital floor blowout fractures with conchal auricular cartilage graft: A report on 14 cases. J. Oral. Maxillofac. Surg., 60 (12): 1413-7, 2002.
7- Ozyazgan I., Baykan H. and Coruh A.: Repair of traumatic orbital wall defects using conchal cartilage. Plast. Reconst. Surg., 117 (4): 1269-76, 2006.

8- Courtney D.J., Thomas S. and Whitfield P.: Isolated orbital blowout fractures: Survey and review. Br. J. Oral. Maxillofac. Surg., 38: 496-503, 2000.

9- Chowdhury K. and Krause G.F.: Selection of materials for orbital floor reconstruction. Arch. Otolaryngol. Head Neck Surg., 124: 1398-401, 1998.

10- Kakibuchi M., Fukuda K., Yamada N., et al.: A simple method for harvesting a thin iliac bone graft for reconstruction of the orbital wall. Plast. Reconstr. Surg., 111: 961-2, 2003.

11- Ozaki W. and Buchman S.R.: Volume maintenance of onlay bone grafts in the craniofacial skeleton: Microarchitecture versus embryologic origin. Plast. Reconstruc. Surg., 102:291-9, 1998.

12- Glassman R.D., Manson P.N., Vanderkolk C.A., et al.: Rigid fixation of internal orbital fractures. Plast. Reconstr. Surg., 86: 1103-11, 1990.

13- Wang K. and Kita Y.: Alloplastic template fixation of blow-out fracture. J. Craniofac. Surg., 13: 510-2, 2002.

14- Sullivan P.K., Rosenstein D.A., Holmes R.E., Craig D. and Manson P.N.: Bone graft reconstruction of the monkey orbital floor with iliac grafts and titanium mesh plates: A histometric study. Plast. Reconstr. Surg., 91: 769-77, 1993.

15- Lee M.J., Kang Y.S., Yang J.Y., Lee do Y., Chung Y.Y. and Rohrich R.J.: Endoscopic transnasal approach for the treatment of medial orbital blow-out fracture: A technique for controlling the fractrured wall with a balloon catheter and Merocel. Plast. Reconstr. Surg., 110: 417-28, 2002.

16- KRAUS M., GATOT A., KAPLAN D. and FLISS D.: Post-traumatic orbital floor reconstruction with nasoseptal cartilage in children; Int. J. Pediatr. Otorhinolaryngol., 64: 187-92, 2002.

17- Constantian M.: Use of auricular cartilage in orbital floor reconstruction; Plast. Reconstr. Surg., 69: 951-5, 1982.

18- Bayat M., Momen-heravi F., Khalilzadeh O., Mirhosseni Z. and Sadeghi-tari A.: Comparison of conchal cartilage graft with nasal septal cartilage graft for reconstruction of orbital floor blowout fractures, Br. J. Oral. Maxillofac. Surg., 48: 617-20, 2010.

19- Talesh K., Babaee S., Vahdati S. and Tabesh-far S.: Effectiveness of a nasoseptal cartilaginous graft for repairing traumatic fractures of the inferior orbital wall; Br. J. Oral. Maxillofac. Surg., 47: 10-3, 2009.

20- Mintz S.M., Ettinger A., Schmakel T., and Gleason M.J.: Contralateral coronoid process bone grafts for orbital floor reconstruction: An anatomic and clinical study. J. Oral. Maxillofac. Surg., 56: 1140-5, 1998.

21- Lin K.Y., Bartlett S.P., Yaremchuk M.J., Fallon M., Grossman R.F. and Whitaker L.A.: The effect of rigid fixation on the survival of onlay bone grafts: An experimental study. Plast. Reconstr. Surg., 86: 449-56, 1990.

22- Zins J.E. and Whitaker L.A.: Membranous versus endochondral bone: Implications for craniofacial reconstruction. Plast. Reconstr. Surg., 72: 778-85, 1983. 